vol. $37-n^{\circ} 1$ et 2 | 2021

Travail et migrations

\title{
Blanchard Melissa, Travail, sexualité et migration. Les commerçantes sénégalaises à Marseille
}

\section{Affaya Rim}

\section{(2) OpenEdition}

Édition électronique

URL : https://journals.openedition.org/remi/18729

DOI : 10.4000/remi. 18729

ISSN : $1777-5418$

Éditeur

Université de Poitiers

Édition imprimée

Pagination : 315-317

ISBN : 979-10-90426-69-6

ISSN : 0765-0752

\section{Référence électronique}

Affaya Rim, « Blanchard Melissa, Travail, sexualité et migration. Les commerçantes sénégalaises à

Marseille ", Revue européenne des migrations internationales [En ligne], vol. 37 - $n^{\circ} 1$ et 2 | 2021, mis en ligne le 25 novembre 2021, consulté le 17 mai 2022. URL : http://journals.openedition.org/remi/18729 ; DOI : https://doi.org/10.4000/remi. 18729

Ce document a été généré automatiquement le 17 mai 2022.

(c) Université de Poitiers 


\title{
Blanchard Melissa, Travail, sexualité et migration. Les commerçantes sénégalaises à Marseille
}

\author{
Affaya Rim
}

\section{RÉFÉRENCE}

Blanchard, Melissa, Travail, sexualité et migration. Les commerçantes sénégalaises à

Marseille. - Aix-en-Provence : Presses Universitaires de Provence, 2018. - 152 p. ISBN :

979-1-03200-181-3

1 Des diverses formes de présence sénégalaise en France, l'on a souvent fait allusion à trois catégories: l'une relative au statut historico-professionnel (tirailleurs dans la guerre, marins) des Sénégalais dans l'histoire militaire et impérialiste de la France, une autre concernant leur circulation économico-religieuse impliquant des mobilités et des réseaux transnationaux et enfin, celle qui sous-tend les logiques de leur immigration postcoloniale et postindustrielle en France (étudiante, regroupement familial) avec son corollaire politique et réflexif, l'intégration. L'ouvrage de Melissa Blanchard, propose en cinq parties une lecture renouvelée des immigrations sénégalaises en France en combinant trois objets de recherche habituellement explorés de manière distincte dans le champ des études migratoires. La tendance qui consistait à mettre les femmes et la famille d'un côté ainsi que le travail et le commerce d'un autre est ici dépassée par une réflexion originale qui retrace l'expérience d'une traversée spatiale, sociale et commerciale au féminin. Marseille, comme point de chute et comme nouvel horizon pour ces femmes, vient ici montrer la compénétration entre le lieu-mémoire (migratoire et commercial) et le processus de renégociation du statut social. En cherchant à éclairer leur rôle en tant que travailleuses et commerçantes, l'auteure souligne la complexité de l'interaction entre l'individualisme, qui s'acquiert dans le contexte migratoire et entrepreneurial, et les obligations communautaires qui continuent à représenter pour elles une sorte d'équilibre individuel par le biais de la 
reconnaissance sociale, l'attachement au pays et le maintien d'une identité culturelle forte.

2 La mise en commun des axes annoncés dans le titre du livre est opportune, car elle contribue à renseigner matériellement la présence genrée des individus qui constituent la communauté marchande des migrations sénégalaises en France et participe ainsi aux travaux féministes qui privilégient « une intersectionnalité située ». Celle-ci implique un regard spécifique sur les rapports de pouvoir multiples qui pèsent sur les trajectoires des femmes, notamment la corrélation entre les tensions juridiques autour du statut de "l'étranger ", l'articulation du productif et du reproductif et celui des «formes » de présence sur lesquelles elles vont s'appuyer pour assurer leur promotion sociale. Des possibilités administratives restreintes qui leur sont offertes se dégagent alors trois figures types de commerçantes : « les femmes en solitaire », « les immigrées du regroupement familial», "les étudiantes». Les catégories qui réglementent les mobilités Sud-Nord se voient ici transformées en situations sociales. Elles deviennent dès lors à la fois des garanties « d'acceptabilité » du projet migratoire féminin dans la société d'origine, mais aussi de « respectabilité » parmi la communauté sénégalaise en terre de migration.

3 Ce que les enquêtées entendent par « les gens derrière » (p. 39) pourrait constituer le fil d'Ariane de ce qui fait la cohérence générale de l'ouvrage : l'imbrication du champ économique et social dans la mise à mal de la contradiction entre individualisme et altruisme. D'apparence irréconciliable, les aspirations à l'indépendance des femmes et leur autonomie subjective épousent, à des degrés variés, l'éthique de solidarité. Réussite personnelle et redistribution des revenus sont corrélées et donnent tout leur sens au parcours des migrantes. Même en étant absents physiquement, les "gens derrière " ne sont pas oubliés si bien que le présent est ordonnancé selon les temporalités d'achats, d'expédition de biens et d'argent et de retour au pays. Hormis le caractère généreux du procédé, le partage des gains représente un puissant indicateur de succès ou d'échec de leurs trajectoires. Ces "présents absents » constituent aussi une autre forme de pression: celle d'une "communauté sans visage» (p. 104) qui empêche les femmes de jouir de leur féminité telle qu'elles la conçoivent au niveau personnel et intime. L'éducation reçue, avec ses prescriptions de chasteté qui entourent la conception de la famille et de la femme, poursuit les migrantes en terre de migration jusqu'à s'interdire, à elles-mêmes et leur descendance, la jouissance d'une liberté sexuelle par peur du «qu'en dira-t-on au Sénégal ? ». Même loin de la famille d'origine, la sentence sociale se réactive par l'autocensure.

Comme en attestent les témoignages, ces « logiques qui cohabitent dans l'existence de chaque migrante» (p.130) ne sont pas exemptes de tensions. La vigilance ethnographique et analytique de l'auteure est démontrée par sa captation de l'univers de sens dans lequel les femmes sont insérées. Commérages, mauvais œil, peur du jugement, entretien de la réputation: autant de mécanismes de contrôle social qui s'avèrent " étouffants » (p. 47), mais dont les femmes s'accommodent au risque de se retrouver isolées. Les compromis qu'elles «fabriquent» témoignent d'un pragmatisme et d'une volonté patiente face au besoin le plus essentiel : la consolidation de leur statut social. Avec un ordre social mis en branle et transformé par la migration, elles vont user de leur pouvoir économique, acquis par le commerce, pour jongler avec les normes, tantôt en s'y conformant, tantôt en les renégociant. Ni fracture ni rupture avec le Sénégal, les récits des migrantes montrent tout le potentiel qu'une approche 
transnationale et féministe des mouvements intraeuropéens et eurafricains pourrait dévoiler sur les multitudes d'objets, d'idées et de services qui sont transportés d'un pays à un autre, et d'un monde social à un autre.

5 À partir de Marseille, cette ville de rassemblement pour les migrants sénégalais et tant d'autres, l'on aperçoit les frontières fluctuantes qui permettent à des petits événements quotidiens de la vie locale de prendre une tout autre dimension. Le dahira, les baptêmes, les mariages, les divers soutiens économiques intracommunautaires, les moments de convivialité, sont tous traversés par la présence d'objets qui circulent à partir du Sénégal, mais aussi d'Italie, de Turquie, d'Espagne, de Chine, de Dubaï ou du Maroc. Des tissus, des sacs à main, des tapis de prière, des bijoux de diverses qualités, des mèches de cheveux et des produits de beauté suivent des itinéraires précis au gré des chaînes relationnelles et migratoires tissées par les migrants sénégalais dans le monde. Une géographie précise avec une «carte des liens» (Weber, 2004 : 38-48) à la disposition des individus qui entraînent dans leurs mouvements des flots d'objets quotidiens et «d'objets-appartenance» (Warnier, 1999) dont les migrantes commerçantes se saisissent pour leur compte.

6 L'ouvrage tire sa valeur de l'originalité de l'hypothèse qui vaut à l'auteure d'articuler avec finesse les interactions, les relations de pouvoir, le dévoilement de l'intimité et des rapports sociaux de sexe. Toutefois, quelques questions sont laissées en suspens. Même si ce n'est pas le propos de l'enquête, le contrôle communautaire semble s'appliquer aux hommes également. Une référence rapide le mentionne sans le mettre en regard avec ce que cela implique pour les couples et les familles. Y a-t-il, par exemple, des modèles d'affranchissement masculin des normes sociales? Par ailleurs, en montrant comment les processus économiques et sociaux se construisent dans la trajectoire commerciale de chacune des migrantes, cette recherche aurait gagné à intégrer une dimension consommatoire. Une vie des objets pourrait être explorée à la lumière des forces capitalistes qui incitent les Sénégalais.es à vouloir se procurer des produits spécifiques. Comment les goûts se construisent-ils et évoluent-ils et selon quelles modalités ? Comment est détourné le capital symbolique des objets en niche lucrative?

7 Malgré ces quelques réserves, la force de ce travail demeure indéniable notamment par l'habileté de l'anthropologue à faire dialoguer de manière équilibrée analyse, description de terrain, récits biographiques et contextualisation. Il s'inscrit dans le champ des études migratoires autant que dans celui de la sociologie de l'action et des études féministes. Il contribue à les enrichir avec sagacité.

\section{BIBLIOGRAPHIE}

Warnier Jean-Pierre (1999) La mondialisation de la culture, Paris, La Découverte.

Weber Serge (2004) De la chaîne migratoire à la migration individuelle des Roumains à Rome, Hommes et Migrations, 1250, pp. 38-48. 


\section{AUTEURS}

\section{AFFAYA RIM}

Doctorante en anthropologie

EHESS 